\title{
Thymine Sensitizes Gram-Negative Pathogens to Antibiotic Killing
}

\author{
Yuan Liu' ${ }^{1,2,3,4 *}$, Kangni Yang ${ }^{1}$, Yuqian Jia ${ }^{1}$, Jingru Shi ${ }^{1}$, Ziwen Tong ${ }^{1}$ and \\ Zhiqiang Wang 1,3,4* \\ ${ }^{1}$ College of Veterinary Medicine, Yangzhou University, Yangzhou, China, ${ }^{2}$ Institute of Comparative Medicine, Yangzhou \\ University, Yangzhou, China, ${ }^{3}$ Jiangsu Co-Innovation Center for Prevention and Control of Important Animal Infectious \\ Diseases and Zoonoses, Yangzhou University, Yangzhou, China, ${ }^{4}$ Joint International Research Laboratory of Agriculture \\ and Agri-Product Safety, The Ministry of Education of China, Yangzhou University, Yangzhou, China
}

OPEN ACCESS

Edited by:

Giuseppantonio Maisetta, University of Pisa, Italy

Reviewed by:

Oana Ciofu,

University of Copenhagen, Denmark

Xuanxian Peng,

Sun Yat-sen University, China

*Correspondence:

Yuan Liu

liuyuan2018@yzu.edu.cn

Zhiqiang Wang

zqwang@yzu.edu.cn

Specialty section:

This article was submitted to

Antimicrobials, Resistance

and Chemotherapy,

a section of the journal

Frontiers in Microbiology

Received: 29 October 2020 Accepted: 04 January 2021

Published: 28 January 2021

Citation:

Liu Y, Yang K, Jia Y, Shi J, Tong Z and Wang $Z$ (2021) Thymine Sensitizes Gram-Negative Pathogens to Antibiotic Killing.

Front. Microbiol. 12:622798. doi: 10.3389/fmicb.2021.622798
Diminished antibiotic susceptibility of bacterial pathogens is an increasingly serious threat to human and animal health. Alternative strategies are required to combat antibiotic refractory bacteria. Bacterial metabolic state has been shown to play a critical role in its susceptibility to antibiotic killing. However, the adjuvant potential of nucleotides in combination with antibiotics to kill Gram-negative pathogens remains unknown. Herein, we found that thymine potentiated ciprofloxacin killing against both sensitive and resistant- $E$. coli in a growth phase-independent manner. Similar promotion effects were also observed for other bactericidal antibiotics, including ampicillin and kanamycin, in the fight against four kinds of Gram-negative bacteria. The mechanisms underlying this finding were that exogenous thymine could upregulate bacterial metabolism including increased TCA cycle and respiration, which thereby promote the production of ATP and ROS. Subsequently, metabolically inactive bacteria were converted to active bacteria and restored its susceptibility to antibiotic killing. In Galleria mellonella infection model, thymine effectively improved ciprofloxacin activity against $E$. coli. Taken together, our results demonstrated that thymine potentiates bactericidal antibiotics activity against Gram-negative pathogens through activating bacterial metabolism, providing a universal strategy to overcome Gram-negative pathogens.

Keywords: antibiotic tolerance, thymine, Gram-negative bacteria, metabolism, antibiotic resistance

\section{INTRODUCTION}

Infectious diseases caused by Gram-positive and Gram-negative bacterial pathogens that are resistant to clinically relevant antibiotics constitute a worldwide public crisis (Boucher et al., 2009; Van Boeckel et al., 2019). According to the Centers for Disease Control and Prevention (CDC) report in 2019, more than 2.8 million infections by antibiotic-resistant bacteria occur in the U.S. each year, and result in more than 35,000 death (CDC., 2019). Compared with Grampositive bacteria, Gram-negative bacteria are harder to treat owing to the highly impermeable outer membrane and limited availability of antibiotics in the clinical setting (Li et al., 2015; Domalaon et al., 2018; Liu et al., 2019a). Thus, there is urgent need to identify novel strategies to combat Gram-negative bacterial infections. In addition to resistance genes-mediated antibiotic resistance, phenotypic resistance mechanisms have been proposed to act a crucial and unappreciated role in the burden of bacterial infections particularly chronic and recurrent infections 
(Meylan et al., 2018; Stokes et al., 2019). Accordingly, this phenotypic resistance could occur in all bacteria, and commonly correlate with the decreased metabolic state of bacteria, including impaired tricarboxylic acid (TCA) cycle, bacterial respiration and bacterial proton motive force (PMF) (Bhargava and Collins, 2015; Stokes et al., 2019). For example, cytochrome oxidase null mutant $(\triangle c y o A \triangle c y d B \triangle a p p B)$ was highly resistant to the lethal effects of bactericidal antibiotics (Lobritz et al., 2015), suggesting that the metabolic state of bacteria is linked to antibiotic efficacy.

By contrast, boosting bacterial metabolism has been shown to effectively improve antibiotic activity against multiple pathogens (Bhargava and Collins, 2015; Liu et al., 2019b, 2020d). For instance, genetically increasing the basal respiration rate of E. coli increased bactericidal antibiotic efficacy over wild-type cells (Lobritz et al., 2015). In addition, specific exogenous metabolites supplementation that can promote bacterial metabolisms were also found to enhance antibiotic efficacy. Supplementation of exogenous alanine and/or glucose significantly enhanced the bactericidal activity of aminoglycoside against spontaneous kanamycin-resistant Edwardsiella tarda via accelerating TCA cycle, the production of nicotinamide adenine dinucleotide $(\mathrm{NADH})$ and proton motive force (PMF) (Peng et al., 2015). Glutamate potentiated aminoglycoside efficacy against Escherichia coli and Edwardsiella tarda through a previously unknown prevalent pathway called pyruvate cycle (the P cycle) (Su et al., 2018). Meanwhile, exogenous glucose increased gentamicin killing against drug-resistant Vibrio alginolyticus through promoting the $\mathrm{P}$ cycle, $\mathrm{NADH}$ and intracellular gentamicin accumulation (Zhang et al., 2019). Moreover, the addition of small thiols such as cysteine can prevent the formation of drug tolerant and drug-resistant cells in cultures (Vilcheze et al., 2017). These examples suggest that exogenous metabolites such as various amino acids have a great potential to restore bacterial susceptibility to antibiotic killing through altering the metabolic state of bacteria.

Nucleotides, one of the important metabolites in organisms, are widely distributed in the human body and have a variety of biological functions, including nucleic acid composition, energy storage, cell metabolism and physiological regulation (Rudolph, 1994; Roy et al., 2016). Recently, an integrated "whitebox" biochemical screening and machine learning approach was developed for revealing causal mechanisms for antibiotic efficacy (Yang et al., 2019). This study showed that purine biosynthesis participates in antibiotic lethality and antibiotic-induced adenine limitation increased ATP demand, which in turn elevated central carbon metabolism and oxygen consumption, and consequently enhanced the bactericidal activity of antibiotics. Furthermore, decreased nucleotides such as adenosine was found in antibiotic-resistant bacteria by GC-MS-based metabolomic profiling (Peng et al., 2015) and elevated pyrimidine was associated with increasing kanamycin killing (Su et al., 2015). Given that the intrinsic correlation between purine biosynthesis and nucleotides, we reasoned that nucleotides may play a positive effect on bacterial metabolism, and further affect antibiotic efficacy.

To that end, we investigated the effect of five nucleotides on the bactericidal activity of antibiotics against Gram-negative bacteria. Consequently, we find that thymine could potentiate multiple bactericidal antibiotics activity against various Gramnegative pathogens through activating bacterial metabolism and respiration, triggering the production of reactive oxygen species (ROS) and thereby improving antibiotic efficacy. These findings suggest that thymine has great potential as a metabolic regulator in assisting antibiotics to eliminate Gram-negative bacteria.

\section{RESULTS}

\section{Thymine Potentiates Ciprofloxacin Killing in a Growth Phase-Independent Manner}

To investigate the effect of nucleotides on antibiotic activity against Gram-negative bacteria, a standard sensitive E. coli ATCC 25922 and multidrug-resistant (MDR) clinical isolate E. coli B2 that is almost resistant to all available antibiotics, were chosen (Liu et al., 2020b; Song et al., 2020). Firstly, we assessed the ciprofloxacin killing (10-fold MIC) against different growth-phase bacteria through counting the surviving percentage of bacteria after co-treatment with five nucleotides. With respect to $E$. coli ATCC 25922, we found that five nucleotides alone had no bactericidal effect on exponentialphase bacteria, and in some extent promoted the stationaryphase bacterial growth. However, the combination of thymine and ciprofloxacin markedly decreased the survival rate of bacteria with above $1-\log _{10}$ in a growth phase-independent manner (Figure 1A). Furthermore, a detailed time-killing curve of E. coli 25922 during $4 \mathrm{~h}$ treatment indicated that purines supplementation slightly undermined the killing activity of ciprofloxacin, whereas pyrimidines particularly thymine drastically potentiated ciprofloxacin activity (Supplementary Figure S1). As for E. coli B2, we found that adenine showed the best potentiation activity with ciprofloxacin with above $2-\log _{10}$ against both exponential phase and stationary phase bacteria. In line with E. coli ATCC 25922, thymine also enhanced the ciprofloxacin killing against E. coli B2 (Figure 1B). In addition, we tested the synergistic activity of ciprofloxacin with five nucleotides against another reference strain E. coli MG1655. As a result, only thymine was found to improve ciprofloxacin activity against E. coli MG1655 (Supplementary Figure S3A), suggesting that thymine-induced potentiation to antibiotic is a general phenomenon across the species and adenine-mediated potentiation is specific to MDR E. coli B2.

Persister cells are a subpopulation of a clonal bacterial population that can survive in high concentrations of antibiotics (Fisher et al., 2017). To assess the synergistic bactericidal activity of nucleotides with antibiotics against persisters, E. coli 25922 (Figure 2A) and E. coli B2 persisters (Figure 2B) were treated with ciprofloxacin (20-fold MIC) in the presence or absence of $10 \mathrm{mM}$ different nucleotides. As a result, thymine remarkably improved ciprofloxacin killing against persister cells, while five nucleotides alone triggered the growth of bacterial persisters (Figure 2). Taken together, these results indicated that adding nucleotides alone during bacterial growth may 

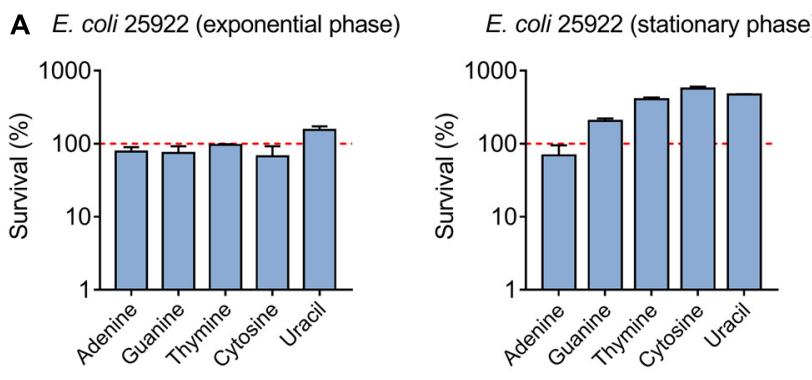

E. coli 25922 (exponential phase)

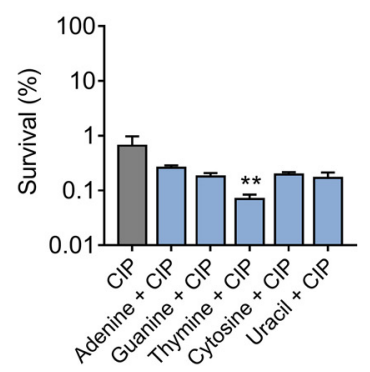

E. coli 25922 (stationary phase)

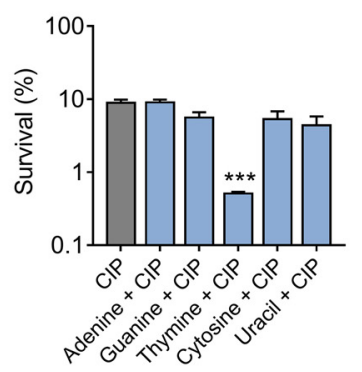

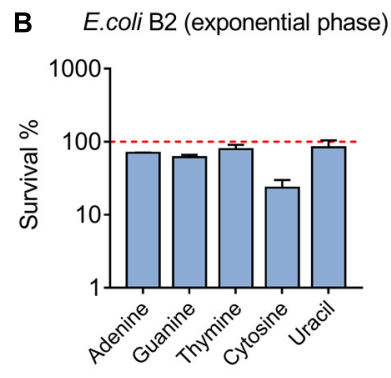

E.coli B2 (exponential phase)

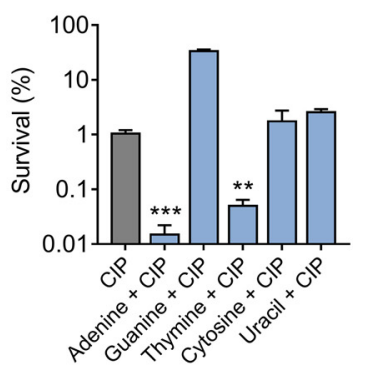

E.coli B2 (stationary phase)

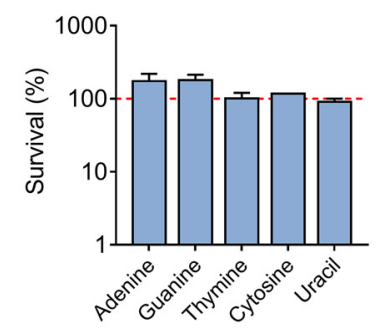

E.coli B2 (stationary phase)

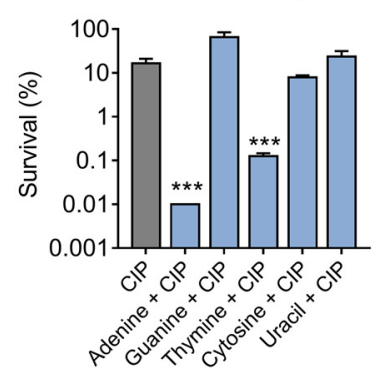

FIGURE 1 | Thymine potentiates ciprofloxacin killing against both sensitive and resistant-E. coli in a growth phase-independent manner. (A) Effect of five nucleotides on ciprofloxacin activity against exponential and stationary phase E. coli ATCC 25922. (B) Adenine and thymine drastically improved ciprofloxacin killing against exponential and stationary phase MDR E. coli B2. All data were obtained in three independent experiments and shown as mean $\pm \mathrm{SD}$. ${ }^{\star} P<0.05,{ }^{\star \star} P<0.01$, ${ }^{\star \star *} P<0.001$, determined by one-way ANOVA.

promote their reproduction, possibly because nucleotides are one of the indispensable substances for bacterial growth. By contrast, some nucleotides particularly thymine in combination with bactericidal antibiotic ciprofloxacin, results in enhanced killing effect against both sensitive and resistant-E. coli in a growth phase-independent manner.

\section{Thymine Enhances the Efficacy of Bactericidal Antibiotics Rather Than Bacteriostatic Antibiotics Against Gram-Negative Pathogens}

Accordingly, bacteriostatic and bactericidal antibiotics result in two fundamentally different phenotypic outcomes, the inhibition of bacterial growth or cell death, respectively (Pankey and Sabath, 2004). They also have differences in perturbing bacterial metabolism. For example, bacterial growth inhibition result from bacteriostatic antibiotics is accompanied with suppressed cellular respiration, whereas cell death from most bactericidal antibiotics is correlated with enhanced respiration (Lobritz et al., 2015). To further validate the potentiating effect of thymine, we tested the survival rate of four kinds of Gramnegative pathogens including $E$. coli 25922, S. enteritidis 13076, A. baumannii 19606, and P. aeruginosa PA14 after combination treatment with bactericidal antibiotics (ampicillin and kanamycin) and bacteriostatic antibiotic such as tetracycline. Consequently, we found that thymine profoundly increased ampicillin and kanamycin activity against multiple pathogens, while no significant alteration for tetracycline (Supplementary Figure S2). These data demonstrated that thymine showed the universal potentiation with bactericidal rather than bacteriostatic antibiotics against a panel of Gram-negative pathogens.

\section{Thymine Sensitizes Gram-Negative Pathogens to Antibiotic Killing Through Upregulating Bacterial Metabolism}

To investigate the underlying mechanisms of action of thymine in potentiating antibiotic efficacy, we firstly determined the MICs of antibiotics in the absence and presence of thymine. As a result, thymine had no effect of the MIC values of antibiotics against both E. coli ATCC 25922 and E. coli B2 (Supplementary Table S3). It has been suggested that antibiotic could target bacterial energy-consuming processes, which result in perturbation of bacterial metabolic homeostasis (Akhova and Tkachenko, 2014). Meanwhile, antibiotic efficacy could be correspondingly enhanced by altering the metabolic state of bacteria (Stokes et al., 2019). Since thymine could not decrease the MIC values of bactericidal antibiotic but still enhance antibiotic killing, we hypothesized that thymine may alter bacterial metabolism and sensitize Gram-negative bacteria to antibiotics. Several studies have been demonstrated that the TCA cycle is a shared mechanism in antibiotic lethality (Kohanski et al., 2007; Meylan et al., 2017). Therefore, we determined the effect of thymine on the bacterial TCA cycle through monitoring the ratio of $\mathrm{NAD}^{+} / \mathrm{NADH}$. Interestingly, the addition of thymine $(10 \mathrm{mM})$ significantly reduced the ratio in stationary-phase E. coli B2 (Figure 3A), indicating that thymine promoted the conversion of $\mathrm{NAD}^{+}$to NADH and thereby possibly accelerated the TCA cycle. Similarly, a decreased ratio of $\mathrm{NAD}^{+} / \mathrm{NADH}$ 

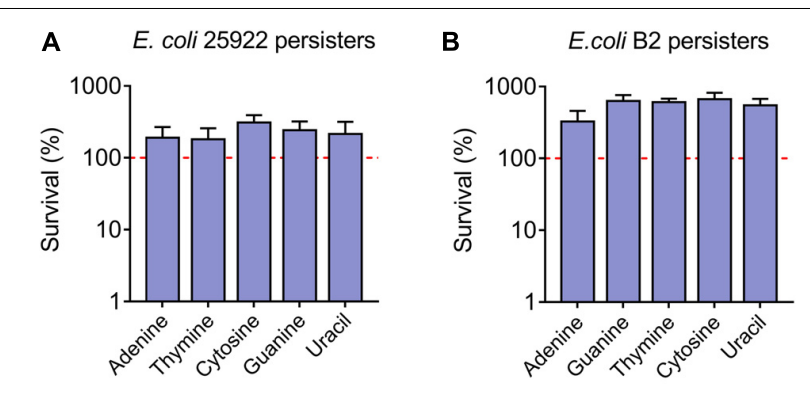

E. coli 25922 persisters
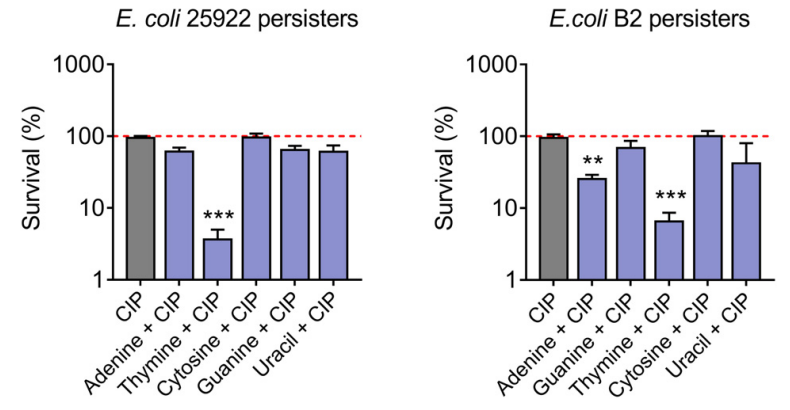

FIGURE 2 | Synergistic bactericidal activity of thymine and ciprofloxacin against $E$. coli persisters. Percentage survival of E. coli 25922 persisters (A) and $E$. coli B2 persisters (B) after exposure to nucleotides $(10 \mathrm{mM})$ or in combination with ciprofloxacin (20-fold MIC, $0.16 \mu \mathrm{g} / \mathrm{mL}$ and $320 \mu \mathrm{g} / \mathrm{mL}$ for E. coli 25922 and E. coli B2 persisters, respectively) for $4 \mathrm{~h}$. All data were obtained in three independent experiments and shown as mean $\pm S D$. ${ }^{\star} P<0.05,{ }^{\star \star} P<0.01,{ }^{\star \star \star} P<0.001$, determined by one-way ANOVA.

in E. coli MG1655 in thymine-treated group was observed (Supplementary Figure S3B). To assess the role of the TCA cycle in the potentiation of thymine, we examined the cell viability in a TCA cycle gene knockout strain E. coli MG1655 ( $\Delta m d h)$ after treatment with ciprofloxacin or in combination with nucleotides. As a consequence, we found that the potentiation of thymine was modestly abolished in knockout strain compared with wild type (Supplementary Figure S3C), implying the crucial role of TCA cycle in the potentiation of thymine.

Considering that the production of $\mathrm{NADH}$ would serve to regulate bacterial electron respiratory chain, thus we next assess whether thymine might elevate bacteria respiration. During $30 \mathrm{~min}$ monitoring, we found that stationary-phase E. coli B2 displayed weak respiration rate, whereas the thymine supplementation remarkedly enhanced bacterial respiration (Figure 3B). Meanwhile, the combination of ciprofloxacin and thymine could further improve bacterial respiration compared with ciprofloxacin alone (Figure 3C). Consistent with our observation, it has been shown that enhanced respiration prevented drug tolerance and drug resistance in Mycobacterium tuberculosis and improved isoniazid killing (Vilcheze et al., 2017). In addition, we determined the intracellular ATP level after exposure to thymine. In agreement with the enhanced respiration, thymine significantly promoted the production of ATP (Figure 3D). This result also explains the profound potentiation of thymine with aminoglycoside antibiotics, which require transmembrane electrochemical gradient for enough cell penetration. These results indicated that the addition of thymine stimulates bacterial respiration and converts tolerant cells to metabolically active cells. Consequently, active bacterial cells would be more sensitive to bactericidal antibiotic killing.

Despite some controversy, ROS has been shown to be critical for antibiotic killing. Although bactericidal antibiotics have well-established mechanisms of action, recent studies revealed that ROS generation is the common consequence of the interaction of antibiotics with their classical targets (Zhao and Drlica, 2014; Acker and Coenye, 2017), which eventually impair synthesis of DNA, lipids and protein. It is wellaccepted that the majority of superoxide generation in E. coli occurs through oxidation of the respiratory electron transport chain driven by oxygen (Kohanski et al., 2007). Therefore, we determined the ROS production in E. coli B2 after exposure to ciprofloxacin or in combination with thymine. Meaningfully, we observed enhanced ROS production in combinational treatment compared with ciprofloxacin alone (Figure 3E), which is in agreement with the finding that enhanced ciprofloxacin killing in the presence of thymine. Consistently, the addition of ROS scavenger thiourea markedly abolished the synergistic bactericidal activity of ciprofloxacin and thymine (Figure 3F), suggesting that ROS production is of importance for potentiation of thymine. Meanwhile, we evaluated pyruvate cycle ([TCA cycle plus phosphoenolpyruvate (PEP)-pyruvate-AcCoA pathway)], antioxidant and electron transport chain (ETC) related gene expression of $E$. coli B2 after treatment with increasing concentrations of thymine (Supplementary Figure S4). Dosedependent increase of the expression of pyruvate cycle genes including icd (isocitrate dehydrogenase), sucA (2-oxoglutarate dehydrogenase), $m d h$ (malate dehydrogenase), fumA (fumarase), $p c k$ (phosphoenolpyruvate carboxykinase), and $p y k A$ (pyruvate kinase II) was observed. In contrast, antioxidant genes such as $\operatorname{sodB}$ (superoxide dismutase) and $a p h C$ (alkyl hydroperoxide reductase) were remarkably decreased under treatment of thymine. Consistent with enhanced ATP levels by thymine, the expression of ETC associated genes including nuoA (NADH-quinone oxidoreductase), cydA (cytochrome oxidase), and $a p t D$ (ATP synthase) were significantly increased. Taken together, these results indicated that exogenous thymine could upregulate bacterial metabolism including increased TCA cycle and respiration, which thereby promote the production of ATP and ROS, and eventually sensitize Gram-negative pathogens to antibiotic killing.

\section{Thymine Supplementation Improves Ciprofloxacin Efficacy in vivo}

Given the attractive potentiation of thymine to bactericidal antibiotics in vitro, we next assessed its synergistic efficacy with ciprofloxacin in a Galleria mellonella infection model (Liu et al., 2020c; Pereira et al., 2020). Galleria mellonella larvae were infected with a lethal dose of E. coli ATCC 25922 or E. coli $\mathrm{B} 2$, then treated with a single dose of ciprofloxacin or thymine monotherapy or their combination. As shown in Figures 4A,B, all larvae after treatment with PBS or thymine died during 3 days, suggesting that thymine monotherapy has no therapeutic effect. 


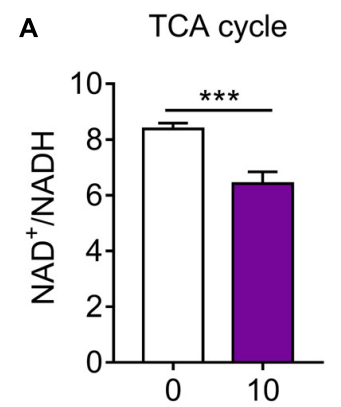

Thymine (mM)

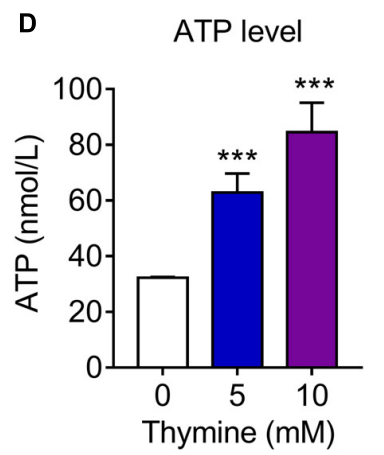

B Bacterial respiration

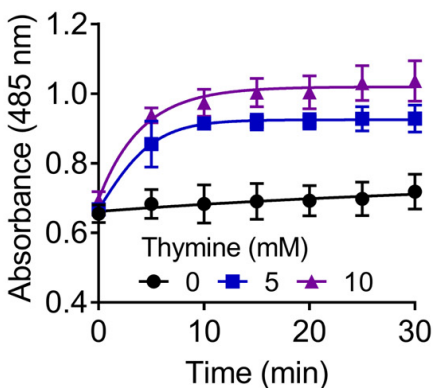

E

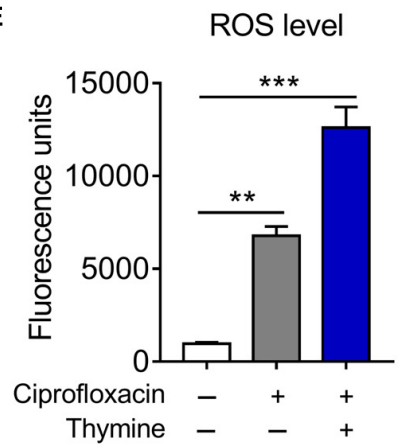

C Bacterial respiration
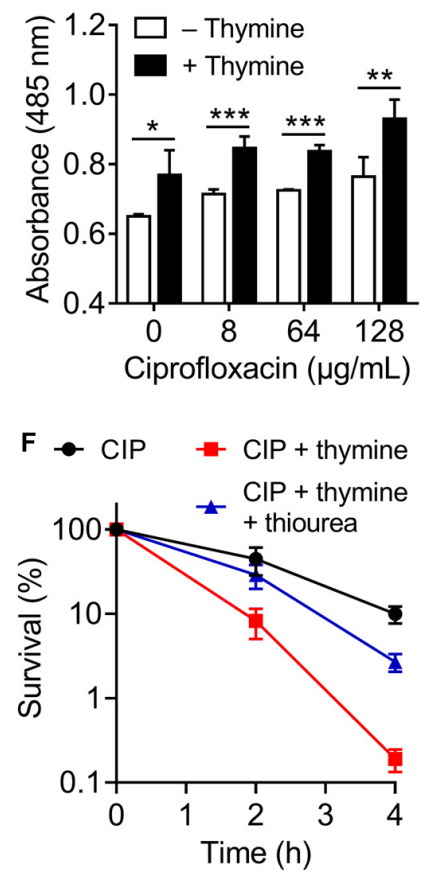

FIGURE 3 | Thymine sensitizes Gram-negative pathogens to antibiotic killing through upregulating bacterial metabolism. (A) Thymine decreased the ratio of $\mathrm{NAD}^{+} / \mathrm{NADH}$, measured by using the water-soluble tetrazolium salt WST-8. (B,C) Thymine significantly enhanced bacterial respiration in the absence (B) or presence (C) of ciprofloxacin through monitoring reduction of iodonitrotetrazolium chloride (INT). (D) Dose-dependent increase of intracellular ATP level by thymine. ATP level was determined based on bioluminescent reaction catalyzed by firefly luciferase. (E) Combination of ciprofloxacin and thymine enhanced ROS production, determined by $2^{\prime}, 7^{\prime}$-dichlorodihydrofluorescein diacetate (DCFH-DA, $10 \mu \mathrm{M}$ ). (F) Addition of ROS scavenger thiourea (100 mM) abolished the synergistic bactericidal activity of ciprofloxacin and thymine. All data were obtained in three independent experiments and shown as mean $\pm \mathrm{SD}$. ${ }^{\star} P<0.05$, ${ }^{\star \star} P<0.01$, ${ }^{\star \star \star} P<0.001$, determined by unpaired $t$-test.

A E. coli ATCC $25922\left(10^{7}\right.$ CFUs)

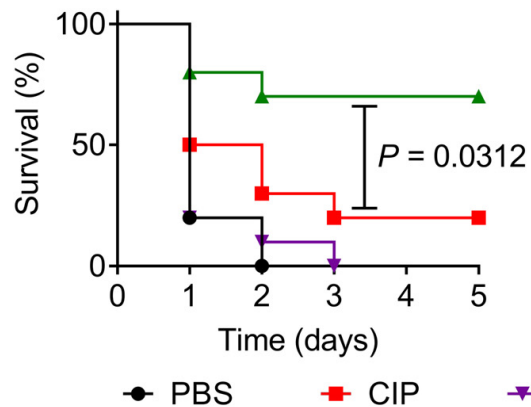

B

E. coli B2 ( $\left.10^{6} \mathrm{CFUs}\right)$

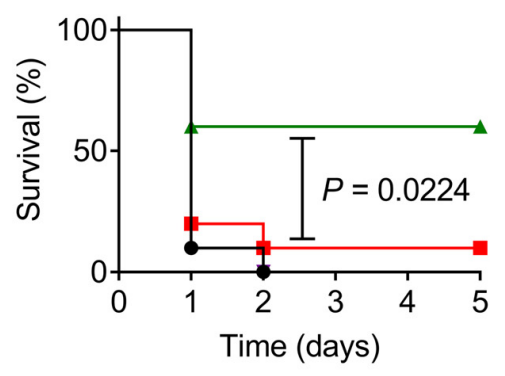

$\star$ CIP + Thymine

FIGURE 4 | Thymine restores ciprofloxacin activity in Galleria mellonella infection model. Galleria mellonella larvae ( $n=10$ per group) was given a lethal inoculum of E. coli ATCC 25922 ( $10^{7}$ CFUs, A) or E. coli B2 (10 6 CFUs, B). After 1-h post-infection, a single dose of PBS, ciprofloxacin (0.08 mg/kg for 25922 or 50 mg/kg for B2), thymine $(10 \mathrm{mg} / \mathrm{kg}$ ) and their combination were given. Survival rates of Galleria mellonella were monitored during 5 days. $P$-values were determined by the log rank (Mantel-Cox) test.

Meanwhile, ciprofloxacin alone showed a weak effect on survival of larvae (only $20 \%$ for 25922 and $10 \%$ for B2). By contrast, combination therapy of ciprofloxacin and thymine significantly increased survival rate of larvae (above 50\%) at 5 days following infection by $E$. coli ATCC $25922(P=0.0312)$ or $E$. coli B2 $(P=0.0224)$.

\section{DISCUSSION}

The growing infections caused by antibiotic resistant bacteria, coupled with a decreasing pipeline of new antibiotics pose a global public health crisis. As such, novel strategies that bypass the need for discovery or design of novel drugs are 
urgently needed to confront antibiotic resistance crisis. There is increasing evidence that the metabolic state of bacteria has an effect on their susceptibility to antibiotic, thus we proposed that antibiotic efficacy may be potentiated by activating bacterial metabolism with exogenous substances (Bhargava and Collins, 2015). Compared with traditional development of antibiotic adjuvants that target specific resistance elements or host immune response, modulating metabolic activity of bacteria offers a generalizable approach to improve bactericidal activity of antibiotics. In particular, such a strategy was first applied to improve aminoglycosides activity against bacterial persisters (Allison et al., 2011). In their study, Allison et al. (2011) showed that the combination of gentamicin and upper glycolysis-related metabolites such as glycose, mannitol and fructose displayed a better eradication of persisters with a CFU reduction of $3-\log$ than gentamicin alone through increasing proton-motive force (PMF) and promoting aminoglycoside uptake. Furthermore, another study also showed that L-lysine potentiates aminoglycosides against $A$. baumannii via the same mechanisms (Deng et al., 2020). These works revealed that the supplementation of specific metabolites may modulate the metabolic state of bacteria and effectively potentiate their susceptibility to bactericidal antibiotics.

Although nucleotides are recognized as one of indispensable metabolites in all organisms, the underlying role of nucleotides in modulating antibiotic efficacy is yet unknown. Thus, in this study, we investigated the effect of five commonly used nucleotides on antibiotic killing against multiple Gram-negative bacteria. As a result, we found that thymine effectively potentiated bactericidal antibiotics killing against both exponential and stationary phase Gram-negative bacteria, as well as persisters and MDR clinical isolates. In addition, adenine only enhanced ciprofloxacin activity on MDR E. coli B2, but not reference strains E. coli ATCC 25922 and MG1655, suggesting that its potentiation is specific to resistant strain E. coli B2. We speculated that the supplementation of adenine may be related to the suppression of specific resistance determinants, however, the accurate modes of action warrants more in-depth investigation. Considering that thymine displayed a universal potentiating effect on various bactericidal antibiotics against multiple Gramnegative bacteria, we hypothesized that thymine may alter the metabolic state of bacteria. To test this, bacterial metabolismrelated factors including TCA cycle, respiration, the production of ROS and ATP were measured. Consequently, we first observed a decrease in $\mathrm{NAD}^{+} / \mathrm{NADH}$ and an increase in respiratory rate of $E$. coli in the presence of thymine, which implies the acceleration of TCA cycle. Then, we found thymine supplementation remarkably enhanced bacterial respiration. Acceleration of the TCA and respiratory rate indicated an increase in the metabolic state of the bacteria, which was also evidenced by the altered ATP level by thymine. It has been acknowledged that the majority of ROS generation in bacteria comes from the oxygen-driven oxidation of the respiratory electron transport chain (Kohanski et al., 2007). Consistently, enhanced ROS production in the combination treatment was found compared with ciprofloxacin alone. ROS has been shown to be a common mechanism of cellular death induced by bactericidal antibiotics (Zhao and Drlica, 2014). For instance, $L$-serine potentiated ofloxacin and moxifloxacin to kill E. coli by increasing the production of $\mathrm{NADH}$, disrupting the $\mathrm{Fe}$ $S$ clusters and thus stimulating endogenous ROS production (Duan et al., 2016). Exogenous alanine or glucose facilitated aminoglycosides killing of antibiotic-resistant E. tarda and $V$. alginolyticus, respectively, via promoting the generation of ROS (Ye et al., 2018; Zhang et al., 2020). These evidences suggest the importance of ROS in the metabolites-enabled potentiation to bactericidal antibiotics.

In summary, our data revealed the huge potential of thymine as a promising metabolism regulator for treatment of bacterial infections caused by Gram-negative bacteria. Specifically, thymine accelerates the bacterial metabolisms particularly respiration, triggers the production of ROS and thereby improves antibiotic efficacy both in vitro and in vivo. These finding inspired that enhancing bacterial metabolism may lead to the discovery of new treatments and improve current antibacterial therapies. However, more studies are still required to systematically assess the in vivo therapeutic potential of the combination of thymine and antibiotics in the clinical setting.

\section{MATERIALS AND METHODS}

\section{Bacterial Strains and Chemical Reagents}

Five reference stain Escherichia coli ATCC 25922, Escherichia coli MG1655, Salmonella enteritidis ATCC 13076, Acinetobacter baumannii ATCC 19609, Pseudomonas aeruginosa PA14, and a multidrug-resistant (MDR) clinical isolate Escherichia coli B2 (Liu et al., 2019c, 2020b; Song et al., 2020) were used in this study. Escherichia coli MG1655 deleted mutant $(\Delta m d h)$ was obtained by homologous recombination mediated by suicide plasmid pLP12. Unless specifically noted, bacteria were grown in Mueller-Hinton broth (MHB) at $37^{\circ} \mathrm{C}$ with $200 \mathrm{rpm}$. To obtain different growth phase bacteria, overnight E. coli were diluted 1:1,000 into $5 \mathrm{~mL}$ fresh $\mathrm{MHB}$, and grown to exponential phase $(4 \mathrm{~h})$ or stationary phase $(8 \mathrm{~h})$. To obtain bacterial persisters, E. coli B2 were grown to latestationary phase for $16 \mathrm{~h}$ at $37^{\circ} \mathrm{C}$ and then pretreated with ciprofloxacin (20-fold MIC, $320 \mu \mathrm{g} / \mathrm{mL}$ ) for $4 \mathrm{~h}$ to kill nonpersistent cells (Morones-Ramirez et al., 2013). Surviving cells were resuspended in $\mathrm{MHB}$, and confirmed as persiters by exposure to higher concentration of ciprofloxacin (40-fold MIC, $640 \mu \mathrm{g} / \mathrm{mL}$ ) for $4 \mathrm{~h}$ and no further CFU reduction was observed. Ciprofloxacin, ampicillin, kanamycin and tetracycline were obtained from China Institute of Veterinary Drug Control. Nucleotides were purchased from TCI chemicals (Shanghai, China).

\section{MIC Measurements}

MICs of different antibiotics were determined using the twofold serially microdilution method according to the Clinical and Laboratory Standards Institute. (2018) guideline (In, 2018). Then, the samples were incubated at $37^{\circ} \mathrm{C}$ for $18 \mathrm{~h}$ and the MIC values were interpreted as the lowest concentrations 
of antibiotics with no visible growth of bacteria. The MICs of each antibiotic for different bacteria have been shown in Supplementary Table S1.

\section{Bactericidal Activity of Antibiotics With or Without Nucleotides}

To assess the effect of different nucleotides on the bactericidal activity of ciprofloxacin against E. coli ATCC 25922 and E. coli B2, exponential phase or stationary phase cells were treated with either ciprofloxacin (10-fold MIC, $0.08 \mu \mathrm{g} / \mathrm{mL}$ for E. coli ATCC 25922 and $160 \mu \mathrm{g} / \mathrm{mL}$ for E. coli B2) or five nucleotides $(10 \mathrm{mM})$ alone or their combination for $4 \mathrm{~h}$. Then, $100 \mu \mathrm{L}$ aliquots were removed, centrifuged at 10,000 g for $5 \mathrm{~min}$ and resuspended in $100 \mu \mathrm{L}$ PBS. Suspensions were serially diluted and spot-plated onto $\mathrm{MH}$ agar plates at $37^{\circ} \mathrm{C}$ overnight to determine $\mathrm{CFUs} / \mathrm{mL}$, and the percentage of surviving cells were calculated. In ROS quenching experiments, stationary phase-E. coli B2 were treated with ciprofloxacin, thymine and thiourea $(100 \mathrm{mM})$ simultaneously. For E. coli 25922 and B2 pesisteres, 20-fold MIC of ciprofloxacin (0.16 $\mu \mathrm{g} / \mathrm{mL}$ and $320 \mu \mathrm{g} / \mathrm{mL}$ for E. coli ATCC 25922 and E. coli B2 persisters) and/or five nucleotides $(10 \mathrm{mM})$ were used. For the synergistic activity of ciprofloxacin and five nucleotides against stationary phase E. coli MG1655 or E. coli MG1655 $(\Delta m d h)$, the synergistic effect of thymine and other three antibiotics (ampicillin, kanamycin and tetracycline) against other three kinds of Gram-negative pathogens (Salmonella enteritidis ATCC13076, Acinetobacter baumannii 19606 and Pseudomonas aeruginosa PA14) in stationary phase, similar protocols were performed as described above.

\section{NAD ${ }^{+} / \mathrm{NADH}$ and Bacterial Respiration Determination}

E. coli $\mathrm{B} 2$ were collected and diluted into $\mathrm{OD}_{600}=0.5$ in $\mathrm{M} 9$. Then, different concentrations of ciprofloxacin $(0,1,5$, and 10 -fold MIC) were added with or without thymine $(10 \mathrm{mM})$. After $4 \mathrm{~h}$ incubation, cell pellets were washed with $0.01 \mathrm{M}$ PBS $(\mathrm{pH}=7.2)$ and re-suspended with $200 \mu \mathrm{L}$ precooled extraction buffer. The lysate was centrifuged at $12,000 \mathrm{~g}$ for $10 \mathrm{~min}$ at $4^{\circ} \mathrm{C}$, and supernatant was measured using $\mathrm{NAD}^{+} / \mathrm{NADH}$ Assay Kit with WST-8 (Beyotime, China).

Effect of thymine on the bacterial respiration was monitored by reduction of iodonitrotetrazolium chloride (INT) (Wenzel et al., 2014). Briefly, E. coli B2 were diluted into $\mathrm{OD}_{600}=0.5$ in $\mathrm{M} 9$, and thymine $(10 \mathrm{mM})$ was added. Subsequently, $1 \mathrm{mM}$ INT and $0.6 \mathrm{mM} \mathrm{NADH}$ were added as substrate and the solution was incubated for $1 \mathrm{~h}$ in the dark. INT reduction was stopped by addition of $5 \%$ trichloroacetic acid. Insoluble formazan was centrifuged at 13,000 $\mathrm{g}$ for $5 \mathrm{~min}$ and extracted with $1 \mathrm{~mL}$ ethanol. Absorbance of the supernatant at $485 \mathrm{~nm}$ was measured during $60 \mathrm{~min}$.

\section{ATP and ROS Measurement}

Intracellular ATP levels of E. coli B2 were determined using an Enhanced ATP Assay Kit (Beyotime, China). E. coli B2 suspension was incubated with thymine $(0,5$, and $10 \mathrm{mM})$ for $1 \mathrm{~h}$. After incubation, bacterial cells were harvested and lysed with lysozyme, and the supernatant was prepared for ATP levels measurement. Detecting solution was added to a 96-well plate and incubated at room temperature for $5 \mathrm{~min}$. Subsequently, the supernatants were added to the wells and its luminescence was measured by Infinite M200 Microplate reader (Tecan). Total ATP levels were calculated from the luminescence signals accordingly.

$2^{\prime}, 7^{\prime}$-dichlorodihydrofluorescein diacetate (DCFH-DA, 10 $\mu \mathrm{M}$ ) were added into $E$. coli B2 suspension (Liu et al., 2020a). After incubated at $37^{\circ} \mathrm{C}$ for $30 \mathrm{~min}, 190 \mu \mathrm{L}$ of probelabeled bacterial cells were added to a 96-well plate and $10 \mu \mathrm{L}$ of ciprofloxacin $(0,1,5$, and 10-fold MIC) without or with thymine $(10 \mathrm{mM})$ were added. After incubation at $37^{\circ} \mathrm{C}$ for $1 \mathrm{~h}$, fluorescence units were immediately measured with the excitation wavelength at $488 \mathrm{~nm}$ and emission wavelength at $525 \mathrm{~nm}$ using an Infinite M200 Microplate reader (Tecan).

\section{Quantitative RT-PCR Analysis}

Exponential phase E. coli B2 were incubated with thymine (0-10 mM) for $4 \mathrm{~h}$. Total RNA was extracted reverse transcribed to cDNA using the PrimeScript ${ }^{\mathrm{TM}} \mathrm{RT}$ reagent Kit with gDNA Eraser (Takara). RT-PCR analysis was performed on a 7500 Fast Real-Time PCR System (Applied Biosystem, CA, United States) using the TB Green qPCR Kit (Takara) with the optimized primers (Supplementary Table S2). Relative quantitative method was applied to calculated the fold changes of mRNA expression relative to the housekeeping gene $r p o C$ (for: GTACGTTCCACATCGGTGGT; rev: CAACCGACTTCACGTTGCTG) (Yang et al., 2020).

\section{Galleria mellonella Infection Model}

Galleria mellonella larvae (purchased from Huiyude Biotech, Tianjin, China) were randomly divided into four groups ( $n=10$ per group) and infected with $10 \mu \mathrm{L}$ of $E$. coli ATCC $25922\left(10^{7}\right.$ CFUs) or E. coli B2 suspension $\left(10^{6}\right.$ CFUs) at the right posterior gastropoda. After 1-h postinfection, larvae were treated with PBS, ciprofloxacin $(0.08 \mathrm{mg} / \mathrm{kg}$ for 25922 or $50 \mathrm{mg} / \mathrm{kg}$ for B2), thymine $(10 \mathrm{mg} / \mathrm{kg})$, or the combination of ciprofloxacin with thymine at the left posterior gastropoda. Survival rates of Galleria mellonella were recorded during 5 days.

\section{DATA AVAILABILITY STATEMENT}

The raw data supporting the conclusions of this article will be made available by the authors, without undue reservation.

\section{AUTHOR CONTRIBUTIONS}

ZW and YL designed this study. YL and KY performed all experiments and wrote the manuscript. YL, KY, YJ, JS, and ZT analyzed the data. All authors have read and agreed to the published version of the manuscript. 


\section{FUNDING}

This work was supported by the National Key Research and Development Program of China (2018YFA0903400), the National Natural Science Foundation of China (32002331), the Natural Science Foundation of Jiangsu Province of China (BK20190893), the Agricultural Science and Technology Independent Innovation Fund of Jiangsu Province [CX(20)3091], the China Postdoctoral Science Foundation (2019M651984), a Project Funded by the Priority Academic Program Development

\section{REFERENCES}

Acker, H. V., and Coenye, T. (2017). The role of reactive oxygen species in antibiotic-mediated killing of bacteria. Trends Microbiol. 25, 456-466. doi: 10.1016/j.tim.2016.12.008

Akhova, A. V., and Tkachenko, A. G. (2014). ATP/ADP alteration as a sign of the oxidative stress development in Escherichia coli cells under antibiotic treatment. FEMS Microbiol. Lett. 353, 69-76. doi: 10.1111/1574-6968.12405

Allison, K. R., Brynildsen, M. P., and Collins, J. J. (2011). Metabolite-enabled eradication of bacterial persisters by aminoglycosides. Nature 473, 216-220. doi: 10.1038/nature10069

Bhargava, P., and Collins, J. J. (2015). Boosting bacterial metabolism to combat antibiotic resistance. Cell Metab. 21, 154-155. doi: 10.1016/j.cmet.2015.01.012

Boucher, H. W., Talbot, G. H., Bradley, J. S., Edwards, J. E., Gilbert, D., Rice, L. B., et al. (2009). Bad bugs, no drugs: no ESKAPE! an update from the infectious diseases society of america. Clin. Infect. Dis. 48, 1-12. doi: 10.1086/595011

CDC. (2019). Antibiotic resistance threats in the United States. Georgia: Centers for Disease Control and Prevention.

Clinical and Laboratory Standards Institute. (2018). Performance standards for antimicrobial susceptibility testing. Wayne, PA: CLSI.

Deng, W., Fu, T., Zhang, Z., Jiang, X., Xie, J., Sun, H., et al. (2020). L-lysine potentiates aminoglycosides against Acinetobacter baumannii via regulation of proton motive force and antibiotics uptake. Emerg. Microb. Infect. 9, 639-650. doi: 10.1080/22221751.2020.1740611

Domalaon, R., Idowu, T., Zhanel, G. G., and Schweizer, F. (2018). Antibiotic hybrids: the next generation of agents and adjuvants against gram-negative pathogens? Clin. Microbiol. Rev. 31:e00077-17. doi: 10.1128/CMR.00077-17

Duan, X., Huang, X., Wang, X., Yan, S., Guo, S., Abdalla, A. E., et al. (2016). L-Serine potentiates fluoroquinolone activity against Escherichia coli by enhancing endogenous reactive oxygen species production. J. Antimicrob. Chemother. 71, 2192-2199. doi: 10.1093/jac/dkw114

Fisher, R. A., Gollan, B., and Helaine, S. (2017). Persistent bacterial infections and persister cells. Nat. Rev. Microbiol. 15:453. doi: 10.1038/nrmicro.2017.42

Kohanski, M. A., Dwyer, D. J., Hayete, B., Lawrence, C. A., and Collins, J. J. (2007). A common mechanism of cellular death induced by bactericidal antibiotics. Cell 130, 797-810. doi: 10.1016/j.cell.2007.06.049

Li, X.-Z., Plésiat, P., and Nikaido, H. (2015). The challenge of efflux-mediated antibiotic resistance in gram-negative bacteria. Clin. Microbiol. Rev. 28, 337418. doi: 10.1128/cmr.00117-14

Liu, Y., Jia, Y., Yang, K., Li, R., Xiao, X., and Wang, Z. (2020a). Anti-HIV agent azidothymidine decreases Tet(X)-mediated bacterial resistance to tigecycline in Escherichia coli. Commun. Biol. 3:162. doi: 10.1038/s42003-020-0877-5

Liu, Y., Jia, Y., Yang, K., Li, R., Xiao, X., Zhu, K., et al. (2020b). Metformin restores tetracyclines susceptibility against multidrug resistant bacteria. $A d v$. Sci. 7:1902227. doi: 10.1002/advs.201902227

Liu, Y., Jia, Y., Yang, K., Tong, Z., Shi, J., Li, R., et al. (2020c). Melatonin overcomes MCR-mediated colistin resistance in gram-negative pathogens. Theranostics 10 , 10697-10711. doi: 10.7150/thno.45951

Liu, Y., Yang, K., Zhang, H., Jia, Y., and Wang, Z. (2020d). Combating antibiotic tolerance through activating bacterial metabolism. Front. Microbiol. 11:577564. doi: 10.3389/fmicb.2020.577564

Liu, Y., Li, R., Xiao, X., and Wang, Z. (2019a). Antibiotic adjuvants: an alternative approach to overcome multi-drug resistant gram-negative bacteria. Crit. Rev. Microbiol. 45, 301-314. doi: 10.1080/1040841x.2019.1599813 of Jiangsu Higher Education Institutions (PAPD), and the Lift Engineering of Young Talents of Jiangsu Association for Science and Technology.

\section{SUPPLEMENTARY MATERIAL}

The Supplementary Material for this article can be found online at: https://www.frontiersin.org/articles/10.3389/fmicb. 2021.622798/full\#supplementary-material

Liu, Y., Li, R., Xiao, X., and Wang, Z. (2019b). Bacterial metabolism-inspired molecules to modulate antibiotic efficacy. J. Antimicrob. Chemother. 74, 34093417. doi: $10.1093 / \mathrm{jac} / \mathrm{dkz} 230$

Liu, Y., Yang, K., Jia, Y., and Wang, Z. (2019c). Repurposing peptidomimetic as potential inhibitor of New Delhi Metallo-beta-lactamases in gramnegative bacteria. ACS Infect. Dis. 5, 2061-2066. doi: 10.1021/acsinfecdis.9b0 0364

Lobritz, M. A., Peter, B., Porter, C. B. M., Arnaud, G., Yang, J. H., Schwarz, E. G., et al. (2015). Antibiotic efficacy is linked to bacterial cellular respiration. Proc. Natl. Acad. Sci. U S A. 112:8173. doi: 10.1073/pnas.1509743112

Meylan, S., Andrews, I. W., and Collins, J. J. (2018). Targeting antibiotic tolerance, pathogen by pathogen. Cell 172, 1228-1238. doi: 10.1016/j.cell.2018.01.037

Meylan, S., Porter, C. B. M., Yang, J. H., Belenky, P., Gutierrez, A., Lobritz, M. A., et al. (2017). Carbon sources tune antibiotic susceptibility in Pseudomonas aeruginosa via tricarboxylic acid cycle control. Cell Chem. Biol. 24, 195-206. doi: 10.1016/j.chembiol.2016.12.015

Morones-Ramirez, J. R., Winkler, J. A., Spina, C. S., and Collins, J. J. (2013). Silver enhances antibiotic activity against gram-negative bacteria. Sci. Transl. Med. 5:190ra181. doi: 10.1126/scitranslmed.3006276

Pankey, G. A., and Sabath, L. D. (2004). Clinical relevance of bacteriostatic versus bactericidal mechanisms of action in the treatment of gram-positive bacterial infections. Clin. Infect. Dis. 38, 864-870. doi: 10.1086/381972

Peng, B., Su, Y.-B., Li, H., Han, Y., Guo, C., Tian, Y.-M., et al. (2015). Exogenous alanine and/or glucose plus kanamycin kills antibiotic-resistant bacteria. Cell Metab. 21, 249-262. doi: 10.1016/j.cmet.2015.01.008

Pereira, M. F., Rossi, C. C., Da Silva, G. C., Rosa, J. N., and Bazzolli, D. M. S. (2020) Galleria mellonella as an infection model: an in-depth look at why it works and practical considerations for successful application. Pathog. Dis. 78:ftaa056. doi: 10.1093/femspd/ftaa056

Roy, B., Depaix, A., Périgaud, C., and Peyrottes, S. (2016). Recent trends in nucleotide synthesis. Chem. Rev. 116, 7854-7897. doi: 10.1021/acs.chemrev. 6b00174

Rudolph, F. B. (1994). The biochemistry and physiology of nucleotides. J. Nutr. 124, 124s-127s. doi: 10.1093/jn/124.suppl_1.124S

Song, M., Liu, Y., Huang, X., Ding, S., Wang, Y., Shen, J., et al. (2020). A broad-spectrum antibiotic adjuvant reverses multidrug-resistant gram-negative pathogens. Nat. Microbiol. 5, 1040-1050. doi: 10.1038/s41564-020-0723-Z

Stokes, J. M., Lopatkin, A. J., Lobritz, M. A., and Collins, J. J. (2019). Bacterial metabolism and antibiotic efficacy. Cell Metab. 30, 251-259. doi: 10.1016/j. cmet.2019.06.009

Su, Y. B., Peng, B., Han, Y., Li, H., and Peng, X. X. (2015). Fructose restores susceptibility of multidrug-resistant Edwardsiella tarda to kanamycin. J. Proteome Res. 14, 1612-1620. doi: 10.1021/pr501285f

Su, Y. B., Peng, B., Li, H., Cheng, Z. X., Zhang, T. T., Zhu, J. X., et al. (2018) Pyruvate cycle increases aminoglycoside efficacy and provides respiratory energy in bacteria. Proc. Natl. Acad. Sci. U S A. 115, E1578-E1587. doi: 10. 1073/pnas.1714645115

Van Boeckel, T. P., Pires, J., Silvester, R., Zhao, C., Song, J., Criscuolo, N. G., et al. (2019). Global trends in antimicrobial resistance in animals in low- and middle-income countries. Science 365:eaaw1944. doi: 10.1126/science.aaw1944

Vilcheze, C., Hartman, T., Weinrick, B., Jain, P., Weisbrod, T. R., Leung, L. W., et al. (2017). Enhanced respiration prevents drug tolerance and drug resistance in Mycobacterium tuberculosis. Proc. Natl. Acad. Sci. U S A. 114, 4495-4500. doi: $10.1073 /$ pnas.1704376114 
Wenzel, M., Chiriac, A. I., Otto, A., Zweytick, D., May, C., Schumacher, C., et al. (2014). Small cationic antimicrobial peptides delocalize peripheral membrane proteins. Proc. Natl. Acad. Sci. U S A. 111, E1409-E1418. doi: 10.1073/pnas. 1319900111

Yang, J. H., Wright, S. N., Hamblin, M., Mccloskey, D., Alcantar, M. A., Schrubbers, L., et al. (2019). A White-box machine learning approach for revealing antibiotic mechanisms of action. Cell 177:e1649. doi: 10.1016/j.cell.2019.04.016

Yang, X., Hashemi, M. M., Andini, N., Li, M. M., Kuang, S., Carroll, K. C., et al. (2020). RNA markers for ultra-rapid molecular antimicrobial susceptibility testing in fluoroquinolone-treated Klebsiella pneumoniae. J. Antimicrob. Chemother. 75, 1747-1755. doi: 10.1093/jac/dkaa078

Ye, J. Z., Su, Y. B., Lin, X. M., Lai, S. S., Li, W. X., Ali, F., et al. (2018). Alanine enhances aminoglycosides-induced ros production as revealed by proteomic analysis. Front. Microbiol. 9:29. doi: 10.3389/fmicb.2018.00029

Zhang, S., Wang, J., Jiang, M., Xu, D., Peng, B., Peng, X. X., et al. (2019). Reduced redox-dependent mechanism and glucose-mediated reversal in gentamicinresistant Vibrio alginolyticus. Environ. Microbiol. 21, 4724-4739. doi: 10.1111/ $1462-2920.14811$
Zhang, S., Yang, M. J., Peng, B., Peng, X. X., and Li, H. (2020). Reduced ROS-mediated antibiotic resistance and its reverting by glucose in Vibrio alginolyticus. Environ. Microbiol. 22, 4367-4380. doi: 10.1111/1462-2920.1 5085

Zhao, X., and Drlica, K. (2014). Reactive oxygen species and the bacterial response to lethal stress. Curr. Opin. Microbiol. 21, 1-6. doi: 10.1016/j.mib.2014. 06.008

Conflict of Interest: The authors declare that the research was conducted in the absence of any commercial or financial relationships that could be construed as a potential conflict of interest.

Copyright (C) 2021 Liu, Yang, Jia, Shi, Tong and Wang. This is an open-access article distributed under the terms of the Creative Commons Attribution License (CC BY). The use, distribution or reproduction in other forums is permitted, provided the original author(s) and the copyright owner(s) are credited and that the original publication in this journal is cited, in accordance with accepted academic practice. No use, distribution or reproduction is permitted which does not comply with these terms. 